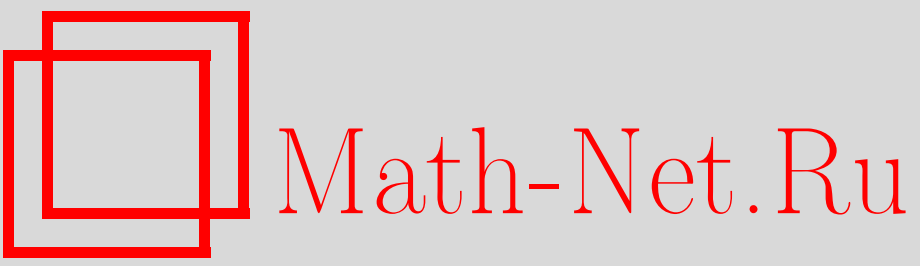

K. Р. Раджагопал, О некоторых нерешенных проблемах нелинейной динамики жидкостей, УМН, 2003, том 58, выпуск 2, 111-122

DOI: https://doi.org/10.4213/rm612

Использование Общероссийского математического портала Math-Net.Ru подразумевает, что вы прочитали и согласны с пользовательским соглашением

http://www.mathnet.ru/rus/agreement

Параметры загрузки:

IP : 54.205 .225 .156

26 апреля 2023 г., 12:26:26 


\title{
О НЕКОТОРЫХ НЕРЕШЕННЫХ ПРОБЛЕМАХ НЕЛИНЕЙНОЙ ДИНАМИКИ ЖИДКОСТЕЙ
}

\author{
К. Р. РАДЖАГОПАЛ
}

В этой статье мы обсуждаем некоторые нерешенные проблемы, касающиеся обобщений жидкостей Навье-Стокса, равно как и других нелинейных жидкостей, которые требуют тщательного изучения. Сначала мы обсудим одно интересное обобщение модели Навье-Стокса, которое, в частности, уместно при описании таких жидкостей, как вода, когда они подвергаются процессам, в которых нормальные напряжения очень велики. Далее мы обсудим моделирование неньютоновских жидкостей вдали от границы занимаемого жидкостью объема. Затем мы обратим внимание на необходимость тщательной ревизии условий, полученных на границах объема жидкости, и, наконец, обсудим моделирование "турбулентных" потоков.

Библиография: 42 названия.

\section{СОДЕРЖАНИЕ}

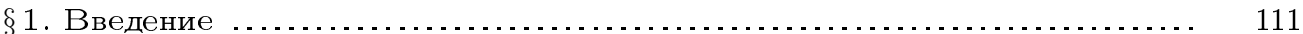

$\S 2$. Модель Навье-Стокса ............................................. 113

§3. Модели жидкостей для процессов, при которых нормальные напря-

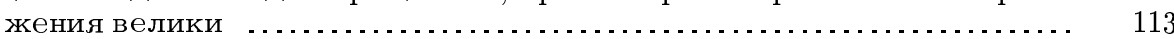

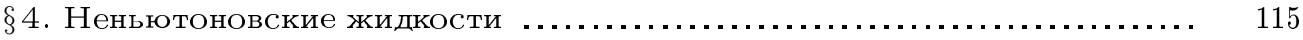

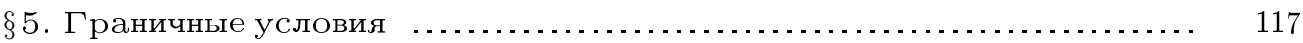

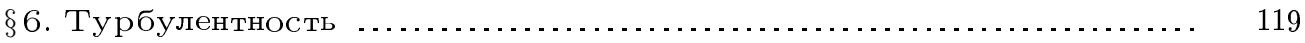

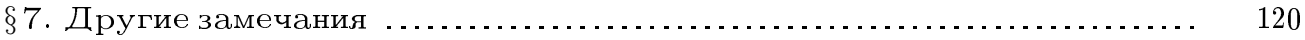

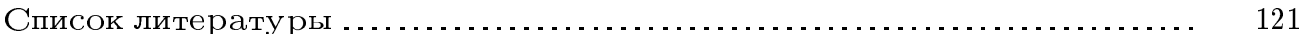

\section{$\S$ 1. Введение}

Для большинства специалистов по гидродинамике динамика жидкостей связана с изучением жидкостей Навье-Стокса. И, вероятно, нет другой такой модели жидкостей, которая подвергалась столь тшательному изучению, как модель Навье-Стокса. Если одномерный вариант данной модели был сформулирован Ньютоном [1], то трехмерные модели были предложены в более поздних работах Навье [2] и Пуассона [3], исходя из несколько иной молекулярной мотивировки, за которой последовали феноменологический вывод Сен-Венана [4] и, наконец, работа Стокса ${ }^{1}$ [5], что окончатель-

\footnotetext{
Работа выполнена при поддержке Национального научного фонда.

${ }^{1}$ Стокс [5] замечает: “Те же самые уравнения были также получены Навье для случая несжимаемых жидкостей (Mém. de l'Académie, t. VI, p. 389), но его аргументация отличается от моей даже больше, чем аргументация Пуассона".
} 
но привело к модели, ньне известной как модель жидкости Навье-Стокса (или модель ньютоновской жидкости). Модель жидкостей Навье-Стокса удерживала центральное место в механике жидкостей на протяжении полутора столетий, и тщательное изучение математиками, физиками и инженерами не дало объяснения ее принщипиальных свойств. Фундаментальные вопросы, касаюшиеся математических свойств уравнений, управляюших потоком таких жидкостей, остаются открытыми, и предсказание поведения таких жидкостей при турбулентных течениях пока не удается физикам и инженерам, хотя большие успехи сделаны в понимании ламинарных течений таких жидкостей, так же как и математических свойств решений уравнений Навье-Стокса (см. монографию О.А. Ладыженской [6]). Определенный прогресс имеется также и в понимании турбулентных потоков жидкостей.

В то время как одни жидкости при некоторых режимах течения описьваются уравнениями Навье-Стокса, существует много других, чье поведение не может быть адекватно описано моделью Навье-Стокса в любом из режимов течения. Эти жидкости принято назвать неньютоновскими. Поведение некоторых жидкостей, таких, как, например, вода, при многих режимах течений достаточно точно описьвается моделью Навье-Стокса, в то время как при других условиях, когда течение является "турбулентным", модель Навье-Стокса неприменима. Таким образом, есть большое количество жидкостей и еше большее количество процессов, которьм эти жидкости подвергаются, для которых модель Навье-Стокса является неприменимой ${ }^{2}$. Эта непригодность, неприменимость или недостаточность в описании поведения реальных жидкостей не может быть отнесена исключительно к моделированию только внутреннего объема жидкостей. Нельзя игнорировать и возможности того, что частично это может происходить от недостаточного понимания процессов, происходяших на границе жидкости, - обстоятельство, которое зачастую недооценивается.

Тензор Коши для жидкостей Навье-Стокса линейно зависит от симметричной части градиента скоростей, и нелинейность в уравнениях Навье-Стокса возникает как следствие вклада сил инерции. В этой небольшой статье мы обратимся к нескольким нерешенным проблемам, касающимся обобщений жидкостей Навье-Стокса, равно как и других нелинейных жидкостей, которые требуют тщательного изучения. Сначала мы обсудим одно интересное обобщение модели Навье-Стокса, которое, в частности, уместно при описании таких жидкостей, как вода, когда они подвергаются процессам, в которых нормальные напряжения очень велики. Далее мы обсудим моделирование неньютоновских жидкостей вдали от гранищы занимаемого жидкостью объема. Затем мы обратим внимание на необходимость тщательной ревизии условий, полученных на границах объема жидкости, и, наконец, обсудим моделирование “турбулентных" потоков.

\footnotetext{
${ }^{2}$ Определяющие соотношения не являются универсально справедливыми для всех тел. Тому факту, что определяющие соотношения выполнены не для всех процессов, часто не придают того значения, которое следовало бы. Однако с точки зрения математического моделирования рассматриваемое тело принято описьвать при помощи определенной модели. Такое соглашение допустимо в том случае, если оно применимо к широкому классу процессов и мы отказываемся от модели в том случае, когда она неприменима. Это означает, что мы должны допустить "ветвление" (бифуркацию) в моделировании тела. Эта точка зрения не нова, такие теории, как пластичность, являются примерами постановок, в которых указанное явление имеет место, хотя в них и не оговаривается точно класс процессов, для которых модель остается справедливой. С другой стороны, слишком громоздко, если и не совсем невозможно, описать весь класс процессов, представляющих интерес, и поэтому считается, что модель, как есть, является точной, а соотношения вьполняются для всех процессов.
} 


\section{§ 2. Модель Навье-Стокса}

Если мы исходим из предположения, что тензор напряжений Коши Т жидкости зависит от плотности $\rho$ и градиента скоростей $\mathbf{L}$, т.е.

$$
\mathbf{T}=\mathbf{f}(\rho, \mathbf{L}),
$$

то условие независимости от системы отсчета (1) означает, что

$$
\mathbf{T}=\mathbf{g}(\rho, \mathbf{D}), \quad \mathbf{D}=\frac{1}{2}\left(\mathbf{L}+\mathbf{L}^{\mathrm{T}}\right) .
$$

Условие изотропности жидкости приводит к соотношению (см. книгу [7])

$$
\mathbf{T}=\alpha_{1} \mathbf{I}+\alpha_{2} \mathbf{D}+\alpha_{3} \mathbf{D}^{2},
$$

где

$$
\begin{aligned}
\alpha_{i} & =\alpha_{i}\left(\rho, \mathrm{I}_{\mathbf{D}}, \mathrm{II}_{\mathbf{D}}, \mathrm{III}_{\mathbf{D}}\right), \\
\mathrm{I}_{\mathbf{D}}=\operatorname{tr} \mathbf{D}, \quad \mathrm{II}_{\mathbf{D}} & =\frac{1}{2}\left[(\operatorname{tr} \mathbf{D})^{2}-\operatorname{tr} \mathbf{D}^{2}\right], \quad \mathrm{III}_{\mathbf{D}}=\operatorname{det} \mathbf{D} .
\end{aligned}
$$

Если потребовать, чтобы тензор напряжений Коши был линеен по $\mathbf{D}$, то мы немедленно получаем, что

$$
\mathbf{T}=-\widehat{\mathbf{p}}(\rho) \mathbf{I}+\lambda(\rho)(\operatorname{tr} \mathbf{D}) \mathbf{I}+2 \mu(\rho) \mathbf{D},
$$

что является моделью для сжимаемой жидкости Навье-Стокса. Далее, предположение несжимаемости жидкости приводит к соотношению для напряжений

$$
\mathbf{T}=-\mathrm{p} \mathbf{I}+2 \mu \mathbf{D}
$$

где - рI есть шаровая часть тензора напряжений. Уравнения (7) являются моделью несжимаемой жидкости Навье-Стокса. Несжимаемая часть (3) имеет вид

$$
\begin{gathered}
\mathbf{T}=-\mathrm{p} \mathbf{I}+\beta_{1} \mathbf{D}+\beta_{2} \mathbf{D}^{2}, \\
\beta_{i}=\beta_{i}\left(\mathrm{II}_{\mathbf{D}}, \mathrm{III}_{\mathbf{D}}\right) .
\end{gathered}
$$

В следуюшем параграфе мы рассмотрим обобщения моделей (6) и (7).

$\S$ 3. Модели жидкостей для процессов, при которых нормальные напряжения велики

При обычных условиях такие жидкости, как вода, можно считать несжимаемыми. Хотя сжимаемость таких жидкостей в ряде процессов учитывать необходимо, сушествуют процессы, при которых, на первьй взгляд, может показаться, что эффекты сжимаемости являются важными, но на самом деле ими можно пренебречь. Таковьми являются ламинарные течения жидкости при очень высоких нормальных напряжениях (давлении). (Примером, для которого указанные условия удовлетворяются, является задача упругогидродинамической смазки.) Если изменение плотности жидкости 
можно считать несущественным (меньше, чем несколько процентов), то в рассматриваемом диапазоне изменения давления вязкость может измениться на несколько порядков ${ }^{3}$, и поэтому такие жидкости можно приближенно считать жидкостями с зависяшей от давления вязкостью. Это значит, что материальньй коэффициент может зависеть от напряжений, которые возникают в напряженном состоянии. Вообще говоря, может оказаться, что коэффициенты материала, характеризующие деформированное тело, могут зависеть от реакции деформации. Обобщение модели (6), которое допускает зависимость от возникаюших при деформации напряжений, есть

$$
\mathbf{T}=-\mathrm{p} \mathbf{I}+\widehat{\alpha}_{2}\left(\mathrm{p}, \mathrm{II}_{\mathbf{D}}, \mathrm{III}_{\mathbf{D}}\right) \mathbf{D}+\widehat{\alpha}_{3}\left(\mathrm{p}, \mathrm{II}_{\mathbf{D}}, \mathrm{III}_{\mathbf{D}}\right) \mathbf{D}^{2}
$$

где р не означает среднее нормальное напряжение (давление), если $\operatorname{tr} \mathbf{D}^{2} \neq 0$. Определяюшее соотношение (10) существенно отличается от определяющих соотношений теории Навье-Стокса или закона Гука, поскольку (10) есть полное определяюшее соотношение. Такие полные реологические теории приводят к значительно более широкому классу решений и оставляют возможность "бифуркации" в определяющих соотношениях. Обобщение (7) принимает вид

$$
\mathbf{T}=-\overline{\mathrm{p}} \mathbf{I}+2 \mu(\overline{\mathrm{p}}) \mathbf{D}
$$

Существует довольно большое количество экспериментальной литературы, касающейся зависимостей вязкости от давления. Многочисленные ссылки на литературу, предшествуюшую 1931 г., можно найти у Бригмана [8]. Ссылки на более новые экспериментальные работы имеются у Хорна и др. [9] и Малека и др. [10]. Жидкости с зависящей от давления вязкостью возникают во многих инженерных задачах. Эксперименты Бейтса и др. [11] и Хаттона и др. [12] являются убедительньми примерами вязкой смазки с зависяшей от скорости сдвига и давления вязкостью. Зависимость от давления является весьма сушественной, поскольку она может быть экспоненциальной (см. посвяшенную газовой смазке книгу Сзери [13], в которой обсуждается важность жидкостей с зависящей от давления вязкостью для приложений в теории смазки). Модели (10) и (11) исследованы не очень детально. В ранних исследованиях Ренарди [14] и Газзола [15] установлены некоторые результаты по разрешимости на малом промежутке времени. Ренарди предполагал, что $\nu(\mathrm{p}) / \mathrm{p} \rightarrow 0$ при $\mathrm{p} \rightarrow \infty$, в то время как экспериментальные данные недвусмысленно демонстрируют обратное. Малек и др. [10] установили существование слабых решений для пространственно-периодических потоков на произвольном промежутке времени. Недавно Франта и др. [16] распространили эти результаты на случай условий Дирихле. Интересное специальное течение, возникающее в технических задачах, было рассмотрено у Хрона и др. [9], [17]. Помимо нескольких этих исследований очень немного внимания уделялось модели (11) и совсем не уделялось более общей модели (10). Нет результатов, касающихся задач обтекания для таких жидкостей, равно как и не проводились исследования устойчивости и бифуркации потоков таких жидкостей. Тщательное изучение решений (10) и (11) оправдано актуальностью этих моделей в технике.

Хотя модели (10) и (11) и являются неньютоновскими, они не относятся к типу моделей, обычно назьваемому неньютоновскими жидкостями. В следуюшем параграфе мы рассмотрим более классические модели ненњютоновских жидкостей.

\footnotetext{
${ }^{3}$ Часто вязкость предполагают зависящей от давления экспоненциально, и на практике она может изменяться на множитель $10^{7}$.
} 


\section{§4. Неньютоновские жидкости}

Отклонение от ньютоновского поведения может происходить самыми разнообразными способами (см. работу автора [18]). Многие жидкости способны уменьшать или увеличивать скорость сдвига (т.е. менять вязкость в зависимости от тензора скоростей деформаций). Другие обладают свойством нелинейной текучести, что не имеет места для жидкостей Навье-Стокса, у которых текучесть линейна. Еще некоторые демонстрируют такие релаксации напряжений, на которые жидкости Навье-Стокса не способны. Еще одно отклонение от ньютоновского поведения - это способность некоторых жидкостей порождать разнищу напряжений для простых профилей течений. ${ }^{4}$

Большинство неньютоновских жидкостей может быть отнесено к одной из следуюших категорий:

i) жидкости дифференциального типа,

ii) жидкости нормального типа,

iii) жидкости интегрального типа.

Мы коротко обсудим каждый из этих классов и укажем некоторые интересные нерешенные математические задачи, касаюшиеся уравнений, описьвающих эти жидкости. Большинство использованных ранее моделей дифференщиального типа могут быть отнесены к классу моделей жидкостей сложности $N$. В таких телах тензор напряжений Коши Т зависит от первых $N$ тензоров Ривлина-Эриксена (см. работу Ривлина и Эриксена [19]) и, возможно, плотности, т.е.

$$
\mathbf{T}=\mathbf{f}\left(\rho, \mathbf{A}_{1}, \ldots, \mathbf{A}_{N}\right)
$$

где

$$
\mathbf{A}_{1}=\mathbf{L}+\mathbf{L}^{\mathrm{T}}, \quad \mathbf{A}_{N}=\frac{d \mathbf{A}_{1}}{d t}+\mathbf{A}_{1} \mathbf{L}+\mathbf{L}^{\mathrm{T}} \mathbf{A}_{1}
$$

$d / d t$ обозначает обычную материальную производную по времени. Тензор Коши для неньютоновских жидкостей сложности $N$ задан соотношением

$$
\mathbf{T}=-\mathrm{p} \mathbf{I}+\mathbf{g}\left(\mathbf{A}_{1}, \ldots, \mathbf{A}_{N}\right) .
$$

Специальньй подкласс жидкостей сложности $N$ - это жидкости порядка $N$ (см. [7]). Несжимаемая жидкость Навье-Стокса является несжимаемой жидкостью порядка 1. Два других типа моделей, принадлежаших классу моделей сложности $N$ и исследованных довольно подробно, это модели сложности 1 вида

$$
\mathbf{T}=-\mathrm{p} \mathbf{I}+\left(\mu\left(\mathbf{A}_{1}\right)\right) \mathbf{A}_{1},
$$

которые включают популярные среди инженеров жидкости степенного типа, а также жидкости второго порядка

$$
\mathbf{T}=-\mathrm{p} \mathbf{I}+\mu \mathbf{A}_{1}+\alpha_{1} \mathbf{A}_{2}+\alpha_{2} \mathbf{A}_{1}^{2}
$$

где $\mu, \alpha_{1}$ и $\alpha_{2}$ предполагаются постоянными.

\footnotetext{
${ }^{4}$ Еще одна характеристика реакции, часто ассоциируемая с неньютоновскими жидкостями, это то, что они не текут до тех пор, пока не будет достигнут некий критический уровень, только после которого жидкость “течет". Такая способность, однако, противоречит самому определению жидкости, а именно, как тела, которое не имеет формы. Поэтому “текучесть", которая предположительно наблюдается, есть следствие того, что шкала времени наблюдения мала по сравнению с естественной шкалой времени, связанной с телом.
} 
Различные модели, относяшиеся к классу, определенному соотношением (14), были исследованы достаточно подробно (см. работы Ладыженской [20], [21], Лионса [22], Малека и др. [23]). Несмотря на столь интенсивные исследования, даже для специального класса жидкостей степенного типа вопросы, касаюшиеся сушествования, единственности и устойчивости решений, остаются открытыми.

Модели, относящиеся к классу (14), не описывают разницу нормальных напряжений, возникающую при простых сдвигах потоков. С другой стороны, модели (15) допускают возникновение разницы нормальных напряжений, и они были использованы для описания процесса плавления жидких кристаллов. Однако модель (15) не может описать уменшшение или увеличение скорости сдвига, и ни одна из моделей (14) или (15) не допускает немедленной упругой реакции или релаксации напряжений. Литература, касающаяся модели (15), может быть весьма запутанной (критический обзор, посвяшенньй этой модели, см. в [24]). Серьезные трудности, связанные с использованием данной модели, возникают из-за наличия в ней тензора Ривлина-Эриксона $\mathbf{A}_{2}$, что приводит к уравнениям движения, которые на порядок выше, чем уравнения Навье-Стокса:

$$
\begin{gathered}
\mu \Delta \mathbf{v}+\alpha_{1}(\Delta \boldsymbol{\omega} \times \mathbf{v})+\alpha_{1} \Delta \mathbf{v}_{t}+\left(\alpha_{1}+\alpha_{2}\right)\left(\mathbf{A}_{1} \Delta \mathbf{v}+2 \operatorname{div}\left[(\operatorname{grad} \mathbf{v})(\operatorname{grad} \mathbf{v})^{\mathrm{T}}\right]\right) \\
-\rho(\boldsymbol{\omega} \times \mathbf{v})-\rho \mathbf{v}_{t}=\operatorname{grad} \mathrm{P}
\end{gathered}
$$

где

$$
\begin{gathered}
\omega=\operatorname{curl} \mathbf{v}, \\
\mathrm{P}=p-\alpha_{1} \mathbf{v} \cdot \Delta \mathbf{v}-\frac{\left(2 \alpha_{1}+\alpha_{2}\right)}{4}\left|\mathbf{A}_{1}\right|^{2}+\frac{\rho}{2}|\mathbf{v}|^{2}+\rho \phi,
\end{gathered}
$$

v обозначает поле скоростей и предполагается, что объемная сила консервативна, т.e. $\mathbf{b}=-\operatorname{grad} \phi$. Поскольку эти уравнения движения на порядок вьше, чем уравнения Навье-Стокса, для них могут понадобиться дополнительные граничные условия. В действительности это как раз тот случай, когда граница является пористой и возможно бесконечное множество решений, которые удовлетворяют условию прилипания на границе (см. [25], [26]). Поэтому интересной открытой проблемой является вопрос о том, как добавить к уравнению (16) граничное условие таким образом, чтобы получить корректно поставленную задачу, обеспечивающую существование, единственность и устойчивость решений. В случае жидкостей порядка $N$ уравнения движения имеют порядок $(N+1)$, и поэтому при $N>1$ снова возникает проблема выбора граничных условий, и чем больше $N$, тем больше условий нужно добавлять.

Схожая ситуация возникает также и при течениях жидкостей нормального типа. Жидкости нормального типа, такие, как жидкости Максвелла, $B$-жидкости Ордлойда, бюргеровские жидкости и т. д., могут описывать релаксацию напряжений, нелинейную текучесть и разницу нормальных напряжений при простых сдвигах течений, но не описьвают увеличение и уменьшение скорости сдвига, в то время как обобщение упомянутых выше моделей эти явления описывают (см. [27]). В случае модели $B$-жидкостей Ордлойда (модель Максвелла получится, если положить коэффищиент равным нулю) тензор напряжений Коши задан соотношениями

$$
\begin{aligned}
\mathbf{T} & =-\mathrm{p} \mathbf{I}+\mathbf{S}, \\
\mathbf{S}+\lambda_{1} \stackrel{\nabla}{\mathbf{S}} & =\mu\left(\mathbf{A}_{1}+\lambda_{2} \stackrel{\nabla}{\mathbf{A}}_{1}\right),
\end{aligned}
$$


где

$$
\stackrel{\nabla}{\mathbf{A}_{1}}=\frac{d \mathbf{A}_{1}}{d t}-\mathbf{L} \mathbf{A}_{1}-\mathbf{A}_{1} \mathbf{L}^{\mathrm{T}} .
$$

Ввиду слагаемого $\frac{d \mathbf{A}_{1}}{d t}$ мы снова сталкиваемся с проблемой, связанной с определением граничных условий в случае пористой границы, если известно, что поток через гранищу не достаточен для того, чтобы гарантировать корректность задачи. ${ }^{5}$ Основные математические проблемы, касаюшиеся сушествования, единственности и т. д., также остаются открытыми для большинства моделей нормального типа.

Большинство математических работ, касающихся моделей жидкостей интегрального типа, ограничиваются моделями линейно-вязких жидкостей. Эти модели не вполне удовлетворяют принципу независимости от системы отсчета. Математическому анализу моделей жидкостей интегрального типа уделялось не так уж много внимания. Популярный класс, K-BKZ-модель (Кэй [28], Бернштейн, Кирсли и Зейпас [29]), был интенсивно исследован численно. Тензор Коши $\mathbf{T}$ задан соотношением

$$
\mathbf{T}=\mathrm{p} \mathbf{I}+2 \int_{-\infty}^{t}\left(\frac{\partial \mathrm{U}}{\partial \mathbf{I}} \mathbf{C}_{t}^{-1}(\tau)-\frac{\partial \mathrm{U}}{\partial \mathrm{II}} \mathbf{C}_{t}(\tau)\right) d \tau
$$

где $\mathrm{U}$ - это функция запасенной энергии, заданная соотношением

$$
\mathrm{U}=\mathrm{U}(\mathrm{I}, \mathrm{II}, t-\tau),
$$

И

$$
\mathrm{I}=\operatorname{tr} \mathbf{C}_{t}(\tau), \quad \mathrm{II}=\operatorname{tr} \mathbf{C}_{t}^{-1}(\tau),
$$

где $\mathbf{C}_{t}(\tau)$ - это тензор левых растяжений. Уравнения движения, соответствуюшие напряжениям, заданньм соотношением (22), приводят к интегрально-дифференциальным уравнениям, и в отличие от моделей дифференциального и нормального типов здесь не возникает трудностей, связанных с определением граничных условий для пористых границ. Однако строгих математических результатов для таких жидкостей установлено немного. Более того, например, в случае полимерной сплавки, для которой используются модели интегрального типа, эксперименты демонстрируют, что происходит проскальзьвание или сдвиг-проскальзывание таких жидкостей на границе, и это проскальзьвание или сдвиг-проскальзьвание сложным образом зависит от условий течения (деформации или нормальных напряжений и т. д.) Это естественньм образом приводит нас к обсуждению граничных условий в механике жидкостей.

\section{§5. Граничные условия}

Недостаточное понимание того, что граничные условия являются определяющими характеристиками, имело очень вредные последствия в механике. Граничные условия определены самой природой материалов, которые предполагаются разделенными границами. Редко эти границы обрисованы “четко". Это очевидно, например, в случае двух помешенных рядом жидкостей, когда молекулы каждой из жидкостей постоянно проникают в объем, занимаемьй другой жидкостью. Основоположники гидродинамики, Навье [2], Пуассон [3] и Стокс [5], ясно осознавали, что граничные условия должны вьводиться. Например, Стокс [5] замечает:

\footnotetext{
${ }^{5}$ Однако модели (19)-(21) можно дать эквивалентную формулировку в виде эволюционных уравнений для кинематического тензора. Для этой модели можно показать замкнутость системы уравнений, снабдив систему начальными условиями.
} 
“Помимо уравнений, которые должны выполняться внутри жидкой массы, необходимо сформулировать уравнения, которые должны выполняться на ее гранищах".

В приведенной цитате ключевыми словами являются "необходимо сформулировать". В действительности Стокс [5] продолжает выводить уравнения. Ранее Навье [2] вьвел уравнения для условия прилипания на границе. Однако Стокс [5] выступил в зашиту использования иных условий в случае, когда такая жидкость, как вода, течет вдоль непроницаемой гранищы, причем это течение достаточно "медленное". Он обосновывал это экспериментами Дю Буа. Однако ранние эксперименты и теоретические разработки (работы Навье, Пуассона, Жиро, Колумба и других) указьвают на большое разнообразие возможностей на границе (см., например, Гольдштейн [30; с. 676-680], [31; с. 7-9], Ламб [32; с. 1213-1214], Дуглас [33; с. 317, 414] для дальнейших ссылок и обсуждения смежных вопросов).

Например, можно допустить свободное проскальзьвание (условие проскальзывания Навье), при котором проскальзывание имеет место при ненулевых касательных напряжениях, а именно (см. [2]),

$$
\mathbf{v} \cdot \boldsymbol{\tau}=-k\left(\mathbf{T}^{\mathrm{T}} \mathbf{n} \cdot \boldsymbol{\tau}\right) \text { на } \Gamma
$$

где $\mathbf{n}$ и $\boldsymbol{\tau}$ обозначают соответственно внешнюю нормаль и касательньй вектор к границе и $k: \Gamma \rightarrow(0, \infty)$.

Многие граничные условия, которые будут использованы ниже, попадают в категорию

$$
\mathbf{v} \cdot \boldsymbol{\tau}=-\mathrm{M}\left(\left|\mathbf{T}^{\mathrm{T}} \mathbf{n} \cdot \boldsymbol{\tau}\right|\right) \frac{\mathbf{T}^{\mathrm{T}} \mathbf{n} \cdot \boldsymbol{\tau}}{|\mathbf{T n} \cdot \boldsymbol{\tau}|} \text { на } \Gamma,
$$

где М является функцией касательных напряжений.

При проскальзывании порогового типа проскальзывание имеет место в случае, когда величина касательного напряжения превосходит некоторое определенное значение. Такие граничные условия часто имеют следуюший вид:

$$
\begin{aligned}
& \left|\mathbf{T}^{\mathrm{T}} \mathbf{n} \cdot \boldsymbol{\tau}\right| \leqslant g \Rightarrow \mathbf{v} \cdot \boldsymbol{\tau}=0 \text { на } \Gamma \\
& \left|\mathbf{T}^{\mathrm{T}} \mathbf{n} \cdot \boldsymbol{\tau}\right|>g \Rightarrow \mathbf{v} \cdot \boldsymbol{\tau} \neq 0 \text { и } \\
& \left(\mathbf{T}^{\mathrm{T}} \mathbf{n} \cdot \boldsymbol{\tau}\right)=-(g+h(|\mathbf{v} \cdot \boldsymbol{\tau}|)) \frac{\mathbf{v} \cdot \boldsymbol{\tau}}{|\mathbf{v} \cdot \boldsymbol{\tau}|} \text { на } \Gamma
\end{aligned}
$$

где $g: \Gamma \rightarrow\left[g_{0}, \infty\right)$ при $g_{0}>0$ и $h: \Gamma \times[0, \infty) \rightarrow[0, \infty)$.

При другом классе условий проскальзьвания, которьй назьвают "проскальзывания обобщенного порогового типа", проскальзывание происходит в случае, когда на граничной поверхности выполняются некоторые соотношения между касательными и нормальными напряжениями. Многие из них имеют вид:

$$
\begin{aligned}
& \left|\mathbf{T}^{\mathrm{T}} \mathbf{n} \cdot \boldsymbol{\tau}\right| \leqslant \mathrm{G}\left(\left|\mathbf{T}^{\mathrm{T}} \mathbf{n} \cdot \mathbf{n}\right|\right) \Rightarrow \mathbf{v} \cdot \boldsymbol{\tau}=0 \text { на } \Gamma \\
& \left|\mathbf{T}^{\mathrm{T}} \mathbf{n} \cdot \boldsymbol{\tau}\right|>\mathrm{G}\left(\left|\mathbf{T}^{\mathrm{T}} \mathbf{n} \cdot \mathbf{n}\right|\right) \Rightarrow \mathbf{v} \cdot \boldsymbol{\tau} \neq 0 \text { и } \\
& \left(\mathbf{T}^{\mathrm{T}} \mathbf{n} \cdot \boldsymbol{\tau}\right)=-\mathrm{N}\left(|\mathbf{v} \cdot \boldsymbol{\tau}|,\left|\mathbf{T}^{\mathrm{T}} \mathbf{n} \cdot \mathbf{n}\right|\right) \frac{\mathbf{v} \cdot \boldsymbol{\tau}}{|\mathbf{v} \cdot \boldsymbol{\tau}|} \text { на } \Gamma,
\end{aligned}
$$

где $\mathrm{N}: \Gamma \times[0, \infty) \rightarrow[0, \infty)$ и $\mathrm{N}$ монотонна. 
Еще один набор граничных условий, которьй представляет интерес, это

$$
\left|\mathbf{T}^{\mathrm{T}} \mathbf{n} \cdot \boldsymbol{\tau}\right| \leqslant \mathrm{g}\left(\left|\mathbf{T}^{\mathrm{T}} \mathbf{n} \cdot \boldsymbol{n}\right|\right), \quad\left(\mathbf{T}^{\mathrm{T}} \mathbf{n} \cdot \boldsymbol{\tau}\right)(\mathbf{v} \cdot \boldsymbol{\tau})=-g\left(\left|\mathbf{T}^{\mathrm{T}} \mathbf{n} \cdot \mathbf{n}\right|\right) \text { на } \Gamma
$$

где $g: \Gamma \rightarrow[0, \infty)$.

Частньй случай этого условия - это

$$
\left|\mathbf{T}^{\mathrm{T}} \mathbf{n} \cdot \boldsymbol{\tau}\right| \leqslant g, \quad\left(\mathbf{T}^{\mathrm{T}} \mathbf{n} \cdot \boldsymbol{\tau}\right)(\mathbf{v} \cdot \boldsymbol{\tau})=-g|\mathbf{v} \cdot \boldsymbol{\tau}| \text { на } \Gamma
$$

- условие, которое использовалось в механике неньютоновских жидкостей.

Другое нестандартное граничное условие, которое заслуживает тщательного изучения, связано с заданием на границе ротора:

$$
(\operatorname{curl} \mathbf{v}) \cdot \tau=w \text { на } \Gamma
$$

где $w$ - заданная функция.

В то время как изучению условий проскальзьвания для жидкостей Навье-Стокса посвящено множество статей, совсем немного строгих математических работ связано с изучением течений неньютоновских жидкостей для различных типов упомянутых вьше условий проскальзьвания и проскальзьвания со сдвигом (единственным исключением является работа Леру [34], в которой рассматриваются течения жидкостей второго рода). Это огорчает, поскольку проскальзьвание представляет значительньй интерес в технике. Подробное обсуждение граничных условий, которые используются в механике жидкостей, а также связанные с ними математические вопросы можно найти в работе Леру и Раджагопала [35].

\section{§ 6. Турбулентность}

В отличие от обсуждавшихся вьше проблем, которые требуют тшательного изучения по причине того, что до сих пор этим проблемам уделялось не так много внимания, как они того заслуживают, турбулентность - это область, которая требует кардинально нового подхода, поскольку многочисленные усилия в ее изучении до сих пор не позволили приблизиться к пониманию загадки этого явления. Библиография, посвященная турбулентности, была бы слишком обширна даже для специального обзора, целиком посвященного этому предмету. Существует огромное количество моделей, которые используются для описания турбулентности: модель вихревой вязкости, модели смешанных длин, модели, полученные как следствие моделирования больших вихрей, статистическая термодинамика, метод перенормированных групп, случайные блуждания и т. д. (Хинзе [36], Маккомб [37], Гэтски и др. [38].)

Здесь мы коротко упомянем методологию, которая до сих пор была не столь активно использована для вьведения довольно перспективных моделей турбулентности. Эта методология основана на схожих чертах, которые наблюдаются в характеристиках течений как при ламинарных течениях неньютоновских жидкостей, так и при турбулентных течениях жидкостей Навье-Стокса (см. работы Ривлина [39], Хуанга и Раджагопала [40]). Вторичные профили, возникающие как для течений неньютоновских жидкостей, так и при турбулентных течениях ньютоновских жидкостей в трубах некруглого сечения, имеют удивительные схожие черты, что наводит на мысль, что определяющие соотношения для их моделей также могут быть похожи. Это значит, что существует сходство в соотношении между напряжениями Рейнольдса и отклонениями скорости при турбулентных потоках и в соотношении между напряжениями и скоростями для ненњютоновских жидкостей. Модель, предложенная Хуангом и 
Раджагопалом [40], основана на упомянутой вьше аналогии и включает в себя как подкласс нелинейную $k$-є-модель, а также модель Йошизава и Нисизима [41], которая была вьведена при помощи методов статистической термодинамики. При моделировании турбулентности этот метод позволяет построить модель, оставаясь в рамках термодинамики. Такие понятия, как зависимость скорости сдвига от скорости, реакция напряжений и возникновение разницы нормальных напряжений при простых сдвигах течений, являются обшими для этих теорий. Также появляется возможность моделировать такие понятия, как задержка турбулентности, при помоши введения невьпуклых энергий, ассоциированных с неньютоновской жидкостью.

Теория, которая может оказаться полезной при моделировании турбулентности, - это современная термомеханическая теория эволюции естественной конфигурации, в которой изменения конфигурации отражают изменения микроструктуры в жидком теле (такая постановка задачи была использована для описания неупругости при развитии структурных дислокаций в твердых телах).

\section{$\S$ 7. Другие замечания}

Проблема, которую не удалось решить для жидкостей Навье-Стокса или для некоторых определенных неньютоновских жидкостей таких, как несжимаемые жидкости второго порядка или максвелловские жидкости, - это проблема определения универсальных течений ${ }^{6}$, которые эти жидкости допускают. Решению проблемы универсальных течений для жидкостей Навье-Стокса были посвящены обширные исследования со стороны Марриса и его соавторов. Также существует ряд работ Трусделла и соавторов, посвященные неньютоновским жидкостям. Решение этих проблем имеет важные последствия (см. [7]).

Большой интерес представляет изучение математических свойств решений уравнений, описывающих течения жидких кристаллов. Эти математические модели содержат направляюшие векторы, а также дополнительньй закон сохранения для них, в котором появляются такие величины, как направляющая тело сила, кинетическая энергия и т. д. K сожалению, величины типа направляюшей силы тела не являются физическими и не могут быть измерены. Экспериментальньй успех этих теорий в ряде частных случаев не должен привести к безоговорочному их принятию, поскольку с философской точки зрения к этим теориям можно предъявить ряд претензий, связанных с необходимостью привлечения дополнительных физических законов сохранения и с практическими трудностями, сопутствуюшими выбору граничных условий для направляюших векторов и т. д. Необходимо развитие глубокой теории анизотропных жидкостей, а затем уже математическое изучение модели. Недавно Раджагопал и Срайниваза [42] разработали теорию анизотропных жидкостей, которая не привлекает дополнительных законов сохранения и не сталкивается с трудностями в выборе дополнительных граничных условий (для направляюшего вектора). Эта теория предлагает способ единого описания при моделировании анизотропных жидкостей. Однако, вероятно, существуют и другие возможности для моделирования анизотропных жидкостей, которые требуют тщательного изучения. Необходимо решить и математические вопросы такие, как существование и единственность решений.

\footnotetext{
${ }^{6}$ Течение является универсальньм, если оно может быть реализовано любой из жидкостей, принадлежащих данному классу. В дополнение к течению, ввиду приведенного вьше обсуждения граничных условий, необходимо фиксировать интересующие нас условия на границе, для которых универсальный класс определяется.
} 
Задачи со свободными границами для неньютоновских жидкостей, проблемы, связанные со смесью жидкостей, аппроксимации, такие, как теория пограничного слоя для неньютоновских жидкостей и турбулентные потоки неньютоновских жидкостей, также являются разделами механики жидкостей, в которых многие вопросы остаются нерешенньми.

\section{СПИСОК ЛИТЕРАТУРЫ}

[1] I. Newton. Philosophiae naturalis principia mathematica, 1687. English transl.: I. Newton. The Mathematical Principles of Natural Philosophy. (The Principia.) / Transl. from the Latin by Andrew Motte in 1729. New York: Prometheus Books, 1995. Рус. пер.: И. Ньютон. Математические начала натуральной философии. М.: Наука, 1989.

[2] C. L. M. H. Navier. Mémoire sur le lois du mouvement des fluides // Mém. Acad. Roy. Sci. Paris. 1823. V. 6. P. 389-416.

[3] S.D. Poisson. Mémoire sur les équations générales de l'équilibre et du mouvement des corps solides élastiques et des fluides // Journal de l'École Polytechnique. 1831. V. 13. P. 1-174.

[4] J. C. Saint-Venant. Note à joindre au Mémoire sur la dynamique des fluides // Mém. Acad. Roy. Sci. Paris. 1843. V. 17. P. 1240-1243.

[5] G. G. Stokes. On the theories of internal friction of fluid in motion, and of the equilibrium and motion of elastic solids // Trans. Cambridge Philos. Soc. 1845. V. 8. P. 287-305.

[6] О. А. Ладыженская. Математические вопросы динамики вязкой несжимаемой жидкости. М.: Наука, 1970.

[7] C. Truesdell, W. Noll. The Non-Linear Field Theories of Mechanics. Berlin: Springer-Verlag, 1965. (Handbuch der Physik. V. III/3.)

[8] P. W. Bridgman. The Physics of High Pressure. New York: McMillan, 1931.

[9] J. Hron, J. Málek, K. R. Rajagopal. Simple flows of fluids with pressure-dependent viscosities // R. Soc. Lond. Proc. Ser. A Math Phys. Eng. Sci. 2001. V. 457. № 2011. P. $1603-1622$.

[10] J. Málek, J. Nečas, K. R. Rajagopal. Global analysis of the flows of fluids with pressure-dependent viscosities // Arch. Ration. Mech. Anal. 2002. V. 165. № 3. P. 243-269.

[11] T.W. Bates, J. A. Williamson, J. A. Spearot, C. K. Murphy. A correlation between engine oil rheology and oil film thickness in engine journal bearing // Society of Automotive Engineers, paper № 860376, 1986.

[12] J. F. Hutton, B. Jones, T. W. Bates. Effects of isotropic pressure on the high temperature high shear viscosity of motor oils // Technical report. Society of Automotive Engineers, 1983.

[13] A.Z. Szeri. Fluid Film Lubrication: Theory and Design. Cambridge: Cambridge Univ. Press, 1998

[14] M. Renardy. Some remarks on the Navier-Stokes equations with a pressure-dependent viscosity // Comm. Partial Differential Equations. 1986. V. 11. № 7. P. 779-793.

[15] F. Gazzola. A note on the evolution Navier-Stokes equations with a pressure-dependent viscosity // Z. Angew. Math. Phys. 1997. V. 48. № 5. P. 760-773.

[16] M. Franta, J. Málek, K. R. Rajagopal. Global existence of weak solutions to the Dirichlet problem for flows of fluids with pressure-dependent viscosity (to appear).

[17] J. Hron, J. Málek, J. Nečas, K.R. Rajagopal. Numerical simulations and global existence of solutions of two dimensional flows of fluids with pressure and shear dependent viscosities // Math. Comput. Simulation (to appear).

[18] K. R. Rajagopal. Mechanics of non-Newtonian fluids // Recent Developments in Theoretical Fluid Mechanics / ed. G. P. Galdi, J. Nečas. Harlow: Longman, 1993. 129-162. (Pitman Res. Notes Math. Ser. V. 291.)

[19] R.S. Rivlin, J. L. Ericksen. Stress-deformation relations for isotropic materials // J. Ration. Mech. Anal. 1955. V. 4. P. 323-425.

[20] О. А. Ладыженская. О новых уравнениях для описания движения вязких несжимаемых жидкостей и разрешимости в целом для них краевых задач // Труды МИАН. 1967. Т. 102. C. $85-104$.

[21] О. А. Ладыженская. О модификациях уравнений Навье-Стокса для болшших градиентов скоростей // Зап. научн. семин. ЛОМИ. 1968. Т. 7. С. 125-154. 
[22] J. L. Lions. Quelques méthodes de résolution des problèmes aux limites non linéaires. Paris: Dunod, 1969

[23] J. Málek, J. Nečas, M. Rokyta, M. Ružižka. Weak and Measure-Valued Solutions to Evolutionary PDEs. London: Chapman \& Hall, 1996. (Appl. Math. Math. Comput. V. 13.)

[24] J. E. Dunn, K. R. Rajagopal. Fluids of differential type: critical review and thermodynamic analysis // Internat. J. Engrg. Sci. 1995. V. 33. № 5. P. 689-729.

[25] K. R. Rajagopal, A.S. Gupta. Remarks on: "On a class of exact-solutions to the equations of motion of a second grade fluid // Internat. J. Engrg. Sci. 1983. V. 21. № 1. P. 61-63.

[26] K. R. Rajagopal, P. N. Kaloni. Some remarks on boundary conditions for flow of fluids of the differential type // Continuum Mechanics and Its Applications (Barnaby, Canada, 1988). New York: Hemisphere Press, 1989. P. 935-942.

[27] K. R. Rajagopal, A. R. Srinivasa. A thermodynamic frame work for rate type fluid models // J. Non-Newton Fluid Mech. 2000. V. 88. № 3. P. 207-227.

[28] A. Kaye. Non-Newtonian flow in incompressible fluids // CoA Note № 134. Cranfield: College of Aeronautics, 1962.

[29] B. Bernstein, E. Kearsley, L. J. Zapas. A study of stress relaxation with finite strain // Trans. Soc. Rheol. 1963. V. 7. P. 391-410.

[30] S. Goldstein. Modern Developments in Fluid Dynamics. V. II. Oxford: Oxford Univ. Press, 1938.

[31] S. Goldstein. Fluid Mechanics in the first half of this century // Ann. Rev. Fluid Mech. 1969. V. 1. P. 1-28.

[32] H. Lamb. Hydrodynamics. New York: Dover, 1932.

[33] R. Dugas. A History of Mechanics. New York: Dover, 1955.

[34] C. Le Roux. Existence and uniqueness of the flow of second-grade fluids with slip boundary conditions // Arch. Ration. Mech. Anal. 1999. V. 148. № 4. P. 309-356.

[35] C. Le Roux, K. R. Rajagopal. A review of non-standard boundary conditions in fluid mechanics (to appear).

[36] J. O. Hinze. Turbulence. New York: McGraw Hill, 1975.

[37] W. D. McComb. The Physics of Fluid Turbulence. Oxford: Oxford Univ. Press, 1991. (Oxford Engrg. Sci. Ser. V. 25.)

[38] T. B. Gatski, M. Y. Hussaini, J. L. Lumley. Simulation and Modeling of Turbulent Flows. New York: Oxford Univ. Press, 1996.

[39] R.S. Rivlin. The relation between the flow of non-Newtonian fluids and turbulent Newtonian fluids // Quart. Appl. Math. 1957. V. 15. P. 212-215.

[40] Y.-N. Huang, K. R. Rajagopal. On a generalized non-linear k- $\epsilon$ model for turbulence that models relaxation effects // Theoret. Comput. Fluid Dynam. 1996. V. 8. № 4. P. 275-288.

[41] A. Yoshizawa, S. Nisizima. A nonequilibrium representation of the turbulent viscosity based on a 2-scale turbulence theory // Phys. Fluids A: Fluid Dynam. 1993. V. 5. № 12 . P. 3302-3304.

[42] K. R. Rajagopal, A. R. Srinivasa. Modeling anisotropic fluids within the framework of bodies with multiple natural configurations // J. Non-Newtonian Fluid Mech. 2001. V. 99. № 2-3. P. 109-124. 